\title{
Cardiac magnetic resonance imaging for the detection of myocardial involvement in granulomatosis with polyangiitis
}

\author{
Alessandro Giollo ${ }^{1,2,4}$ (C) . Raluca B. Dumitru ${ }^{1,2} \cdot$ Peter P. Swoboda ${ }^{3} \cdot$ Sven Plein $^{1,3} \cdot$ John P. Greenwood ${ }^{1,3}$. \\ Maya H. Buch ${ }^{2,5,6}$. Jacqueline Andrews ${ }^{1}$
}

Received: 23 March 2020 / Accepted: 5 October 2020 / Published online: 14 October 2020

(c) The Author(s) 2020

\begin{abstract}
The prevalence of undiagnosed cardiac involvement in granulomatosis with polyangiitis (GPA) is unknown. In this prospective study we investigated the utility of cardiovascular magnetic resonance (CMR) to identify myocardial abnormalities in GPA and their correlation with disease phenotype. Twenty-six patients with GPA and no cardiovascular disease or diabetes mellitus underwent contrast-enhanced CMR, including late gadolinium-enhancement (LGE), T1-mapping for native T1 and extra-cellular volume (ECV) quantification for assessment of myocardial fibrosis, cine imaging and tissue tagging for assessment of left ventricular (LV) function. Twenty-five healthy volunteers (HV) with comparable age, sex, BMI and arterial blood pressure served as controls. Patients with GPA had similar cardiovascular risk profile to HV. A focal, non-ischaemic LGE pattern of fibrosis was detected in $24 \%$ of patients and no controls $(\mathrm{p}=0.010)$. Patients with myocardial LGE were less frequently PR3 ANCA (7\% vs 93\%, $\mathrm{p}=0.007)$, and had involvement of the lower respiratory tract and skin. LGE scar mass was higher in patients presenting with renal involvement. Native T1 and ECV were higher in patients with GPA than HV; ECV was higher in those with relapsing disease, and native T1 was inversely associated with PR3 ANCA $(\beta=-0.664$, $\mathrm{p}=0.001$ ). Peak systolic strain was slightly reduced in GPA compared to controls; LV ejection function was inversely correlated with disease duration $(\beta=-0.454, p=0.026$ ). Patients with GPA have significant myocardial abnormalities on CMR. ANCA, systemic involvement and disease severity were associated with myocardial fibrosis. CMR could be a useful tool for risk stratification of myocardial involvement in GPA.
\end{abstract}

Keywords Vasculitis · Granulomatosis with polyangiitis · Cardiovascular disease · CMR · ANCA · Myocardial fibrosis · LGE

\section{Introduction}

Alessandro Giollo and Raluca Dumitru contributed equally to this work.

Electronic supplementary material The online version of this article (https://doi.org/10.1007/s10554-020-02066-2) contains supplementary material, which is available to authorized users.

Alessandro Giollo

alessandro.giollo@univr.it

1 NIHR Leeds Biomedical Research Centre and Clinical Research Facility, Leeds Teaching Hospitals NHS Trust, Leeds, UK

2 Leeds Institute of Rheumatic and Musculoskeletal Medicine, University of Leeds, Leeds, UK

3 Leeds Institute of Cardiovascular and Metabolic Medicine, University of Leeds, Leeds, UK
Granulomatosis with polyangiitis (GPA), a systemic inflammatory disease, is an ANCA-associated small-vessel vasculitides (AAV) [1] with significant morbidity and mortality $[2,3]$. Recent epidemiological studies have highlighted the increased frequency of cardiovascular $(\mathrm{CV})$ events in

4 Rheumatology Section, Department of Medicine, University of Verona, Policlinico G.B. Rossi 10, 37134 Verona, Italy

5 Centre for Musculoskeletal Research, University of Manchester, Manchester, UK

6 NIHR Manchester Biomedical Research Centre, Manchester Teaching Hospitals NHS Trust, Manchester, UK 
patients with AAV and GPA [4-6], with resulting higher death rates from CV disease (CVD) [7, 8].

The increased morbidity and mortality due to CVD in patients with immune-mediated and inflammatory diseases (IMID) is thought primarily to be due to accelerated atherosclerosis [9]. However, primary myocardial involvement due to inflammation unrelated to typical atherosclerotic processes has been described in patients with systemic vasculitides [10-14], despite modern era immunosuppression [15].

Despite clear evidence of adverse CV outcomes in GPA, the underlying pathogenesis for cardiovascular involvement in GPA is poorly understood. Inflammatory myocardial involvement is probably underestimated as it is often not clinically looked for or detected until a CV event occurs. A retrospective echocardiography study revealed CV abnormalities related to GPA in up to $36 \%$ of patients [16]. Although the impact of subclinical CVD in GPA is not yet known, in the general population it has been associated with an increased risk of CV events in large population studies $[17,18]$.

Cardiovascular magnetic resonance (CMR) is a non-invasive imaging technique that is increasingly used to detect CVD in patients with IMID [19] with clear advantages over echocardiography. In particular, CMR is highly accurate and reproducible for the evaluation of left ventricular (LV) volumes, function and mass, and for advanced myocardial tissue characterization [20]. In contrast to echocardiography, CMR imaging with late gadolinium enhancement (LGE) has the ability to detect focal myocardial fibrosis [21]. Myocardial T1 mapping methods with calculation of extracellular volume fraction (ECV) can be useful in detecting oedema and early subclinical, diffuse myocardial fibrosis, with good correlation to histological findings of myocardial fibrosis in various clinical contexts [22, 23].

Although preliminary reports suggest that CMR is valuable in AAV [24, 25], a systematic assessment of GPA patients with CMR imaging has not been performed. The primary objective of this single-centre, observational, prospective study was to determine the prevalence and pattern of subclinical cardiac involvement in GPA as detected by CMR imaging; the secondary objective was to describe which GPA disease characteristics were associated with cardiac abnormalities detected by CMR.

\section{Methods}

\section{Study design}

This study was a single-centre, observational, cross-sectional feasibility study. Consecutive patients between 18 and 80 years attending the Vasculitis clinic at Leeds Teaching
Hospitals NHS Trust (LTHT) were approached to enter the study. In addition to a comprehensive clinical evaluation at baseline, participants underwent contrast-enhanced CMR. All patients gave their written, informed consent to take part in the study with approval of the National Research Ethics Service (10/H1306/88).

\section{Study population}

All patients invited to participate met the 2012 revised Chapel Hill Consensus nomenclature for vasculitis [26]. Selection criteria was age $18-80$, complete remission with a Birmingham Vasculitis Activity Score (BVAS) $<0$ following induction therapy and no prior diagnosis of CVD or diabetes mellitus to minimise the possibility of subclinical CVD occurring due to multimorbidity. Controls were identified from a previously recruited pool of 80 healthy volunteers (HV) with no diabetes mellitus (DM) and no known CVD with their imaging assessments recorded using the same CMR scanner as the vasculitis subjects. The controls were frequency-matched to GPA patients according to age, sex, BMI and mean arterial pressure (MAP). All participants (both vasculitis subjects and HV) were excluded if they had $\mathrm{DM}$, uncontrolled arterial hypertension or a prior diagnosis of CVD including: heart failure (HF), coronary artery disease (CAD), cerebrovascular accident (CVA), valve disease, and peripheral arterial disease (PAD).

\section{Clinical data}

Comprehensive demographic and clinical data were collected including the following CV risk factors: Hypertension was defined as a systolic blood pressure (SBP) of at least $140 \mathrm{mmHg}$ and/or a diastolic blood pressure (DBP) of at least $90 \mathrm{mmHg}$, and/or pharmacologically treated blood pressure of unknown cause. Obesity was identified as a BMI $\geq 30 \mathrm{~kg} / \mathrm{m}^{2}$. Dyslipidaemia was defined as levels of total serum cholesterol (TC) above $190 \mathrm{mg} / \mathrm{dl}$ and/or triglycerides (TG) above $150 \mathrm{mg} / \mathrm{dl}$, or pharmacologically treated high lipid serum levels. To assess renal function, we considered the glomerular filtration rate (GFR) estimated with the chronic kidney disease-epidemiology (CKD-EPI) equation.

GPA assessment was recorded as disease phenotype (organ involvement, disease duration, ANCA status, number of relapses), past and current medications, disease activity assessed by BVAS, 3rd version [27] and damage assessed by the Vasculitis Damage Index (VDI) [28].

Disease relapse was defined as reappearance or worsening of vasculitis symptoms requiring an increase of the current treatment or introduction of additional immunosuppressive medication. 


\section{Biochemical assessment}

Blood samples were collected in close proximity to the day of CMR assessment. The following measurements were performed: full blood counts (FBC), creatinine (P-Cr), blood urea nitrogen (BUN), anti-neutrophil cytoplasm antibodies (ANCA), C-reactive protein (CRP), and erythrocyte sedimentation rate (ESR).

\section{CMR protocol and analysis}

CMR studies were performed on a 3T Philips Achieva MR system. Trained CMR radiographers directed the scan, and image analysis was performed in accordance with recognised reporting standards [29] and using established and validated protocols [30]. The full CMR protocol included resting wall motion and LV function, tissue tagging, aortic distensibility, LGE, native and post contrast T1 mapping for ECV quantification. Native T1 and ECV were measured in areas without scar on LGE. Comprehensive and detailed descriptions of each analysis and their interpretation are available in the on-line supplementary file. LGE analysis was performed by two independent assessors (RBD, JG) and quantified using the Full Width Half Maximum (FWHM) technique. Aortic distensibility was defined as decreased if below the 5 th centile of a reference population [31]. Normal ranges for ECV was defined as increased if above the upper limit of a reference range previously reported [32].

\section{Statistical analysis}

As a feasibility study, formal power calculation for determining sample size was not indicated. Descriptive statistics are reported. Continuous data are represented by median [25th, 75th percentile]. Categorical data are described as absolute number (percentage). Between groups comparisons of continuous variables, including differences between GPA patients and heathy volunteers, were performed with the Mann-Whitney $\mathrm{U}$ test. Associations between dichotomous variables were assessed by Chi squared test or Fisher's exact test where appropriate. The strength of the association between disease characteristics, CV risk factors and continuous CMR measures were assessed by the Spearman's rho (r) coefficients. The statistical package IBM SPSS Statistics for Windows, Version 20.0 (Armonk, New York, USA) was used for the matching procedure and statistical analysis. Statistical significance was considered for a $\mathrm{P}$ value of 0.05 or less.

\section{Results}

\section{Patient disposition}

Sixty-four patients were consecutively approached between October 2017 and April 2018 from which 32 patients consented to enter the study and 26 patients proceeded to CMR assessment (patient flow pathway is illustrated in Fig. 1). One patient did not receive gadolinium-based contrast due to their inability to tolerate the intravenous (IV) infusion.

\section{Baseline characteristics of study participants}

The baseline patient characteristics are reported in Table 1 . Median age of GPA patients was 58 years and all but one of the patients were white British. All patients had established disease as per study protocol, three-quarters were ANCA positive, and almost all received either CYC or RTX for induction of remission. ENT, pulmonary and renal involvement was frequent (over 60\%), and the majority had suffered at least one disease relapse.

With regards to CV profile (Table 2), 19/26 (73\%) patients had at least one (median $1[0,3]$ ) traditional CV risk factor. Approximately half of the study population was taking CV medications. As per protocol, heathy volunteers did not differ significantly from patients with GPA when compared for age, BMI, BP and the male-to-female ratio.

\section{Comparison of CMR measures between GPA subjects and heathy volunteers}

Compared to heathy volunteers, GPA patients had smaller chamber volumes and decreased LV mass. Native T1 and ECV were significantly higher in GPA than controls $(6 / 25$ patients had increased ECV, no HV had increased ECV). LGE was detected in 8/25 (32\%) GPA patients and in no controls. No GPA patient had LGE in a pattern of enhancement suggesting ischaemic-mediated myocardial damage. A focal pattern of fibrosis was seen in all GPA patients with LGE. LGE most commonly involved the basal LV and lateral segments and was located in the midwall to subepicardial layer in 6/8 (75\%) patients (Table 3; Fig. 2). Two patients had evidence of scarring uniquely in the inferior wall and right ventricle insertion point (RVIP). However, the pathogenesis and clinical relevance of LGE at RVIP remains poorly understood, and is generally believed to be a nonspecific finding in CMR studies, although a few reports suggest that RVIP-LGE may be related to pulmonary hypertension [33-36]. Hence, when subsequently we analysed correlations and associations of clinical variables with LGE, we chose to exclude the two patients with isolated RVIP-LGE, 
Fig. 1 Flow chart of GPA patients participating in the study

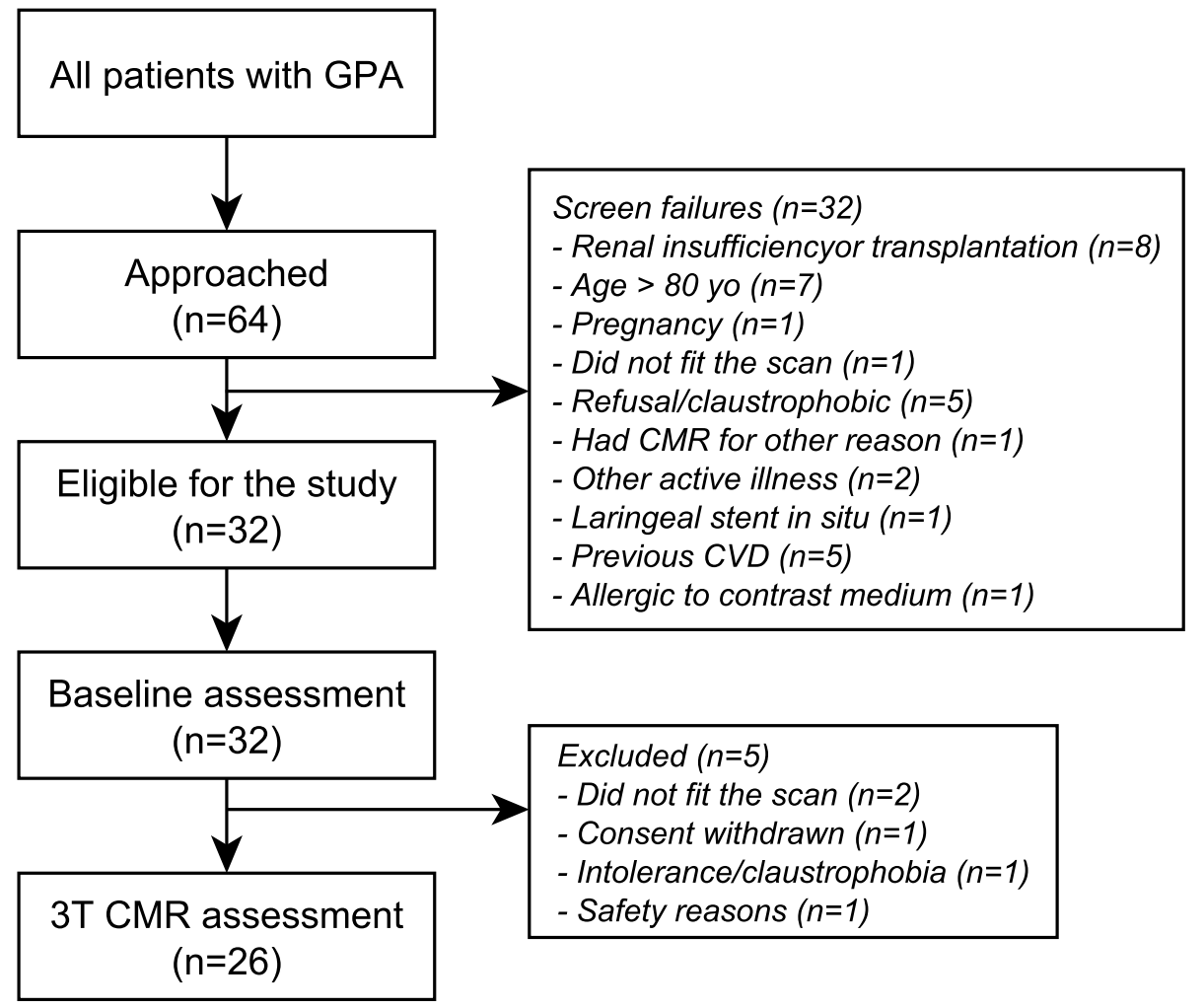

as we believed it had a low likelihood to represent a distinct pattern of myocardial fibrosis in our patients with GPA. There was no difference in LV EF between patients with GPA and heathy volunteers (Table 3), and values for strain were within normal range both in patients and healthy volunteers.

\section{Relationship between traditional CV risk factors and CMR measures}

In the whole study population, diastolic BP was associated with LVEDV index independent of age and sex $(\beta=-0.29$, $\mathrm{p}=0.042$ ). In patients with GPA, the descending aorta distensibility was decreased compared to reference values in $19 / 26$ (73\%; $100 \%$ of females and $50 \%$ of males) and correlated inversely with age $(\mathrm{r}=-0.532, \mathrm{p}=0.005)$, LGE scar mass $(r=-0.810, p=0.015), \operatorname{MAP}(r=-0.519, p=0.007)$ and SBP $(r=-0.650, p<0.001)$. We did not find significant associations between other $\mathrm{CMR}$ measures and $\mathrm{CV}$ risk factors.

\section{Associations of CMR measures with disease characteristics of GPA}

LVESV index was associated with PR3 ANCA status $\left(23 \mathrm{ml} / \mathrm{m}^{2}[21,34]\right.$ in PR3 ANCA negative vs $31 \mathrm{ml} / \mathrm{m}^{2}$ $(26,38)$ in PR3 ANCA positive patients, $p=0.032)$ and correlated with disease duration $(r=0.420, p=0.033)$.
LVEF was positively correlated to disease duration as well $(\mathrm{r}=-0.429, \mathrm{p}=0.029)$. Native $\mathrm{T} 1$ was associated with PR3 ANCA status (1268 ms [1248, 1337]) in PR3 ANCA negative vs $1212 \mathrm{~ms}[1196,1236]$ in PR3 ANCA positive patients, $\mathrm{p}<0.001)$. ECV was significantly higher in GPA patients with relapsing disease $(25.6 \%$ [24.2, 29.8] vs $24.1 \%$ [22.1, 25.8], $\mathrm{p}=0.037)$. All results via univariate analysis.

\section{Characteristics of GPA patients with and without LGE}

Patients with myocardial LGE (LGE+) were more frequently PR3 ANCA negative (93\% vs 7\%, p=0.007), and more likely to have involvement of the lower respiratory tract $(75 \%$ vs $25 \%, \mathrm{p}=0.097)$ and skin $(63 \%$ vs $38 \%$, $\mathrm{p}=0.087)$. No significant differences were noted in previous or current immune-suppressive regimes, although there was a trend to less frequent use of RTX in LGE + patients (25\% vs $75 \%, \mathrm{p}=0.081)$. Native $\mathrm{T} 1$ values were higher in LGE + patients: 1259 ms [1234, 1284] vs $1220 \mathrm{~ms}$ [1197, $1270], p=0.023$, and ECV was only modestly increased in this group. Patients with GPA and LGE + had a significantly higher LV mass/LVEDV ratio: 0.64 [0.60, 0.67] vs 0.52 [0.47, 0.63], $\mathrm{p}=0.043$. The LGE scar mass was associated with renal involvement: $0.82 \mathrm{~g}[0.80,1.09]$ vs $2.98 \mathrm{~g}$ [1.58, 4.51], $\mathrm{p}=0.036$. 
Table 1 Disease characteristics of patients with AAV

\begin{tabular}{ll}
\hline Disease characteristics & GPA patients $(\mathrm{n}=26)$ \\
\hline Disease duration (year) & $8(2,12)$ \\
Biopsy obtained [n(\%)] & $20(77)$ \\
ANCA-positive at diagnosis [n (\%)] & $20(77)$ \\
IFI & $22(85)$ \\
ANCA PR3 & $15(58)$ \\
ANCA MPO & $6(23)$ \\
Organ involvement [n (\%)] & \\
Ear, nose, throat & $22(85)$ \\
Pulmonary & $13(50)$ \\
Renal & $13(50)$ \\
Musculoskeletal & $12(46)$ \\
Cutaneous & $9(35)$ \\
Ophthalmological & $8(31)$ \\
Neurological & $7(27)$ \\
Gastrointestinal & $0(0)$ \\
Induction treatment [n (\%)] & \\
Cyclophosphamide & $18(69)$ \\
Rituximab & $5(19)$ \\
Other & $3(12)$ \\
Relapsing disease [n (\%)] & $17(65)$ \\
Last relapse (months) & $42(10,91)$ \\
Current treatments [n $(\%)]$ & $26(100)$ \\
Methotrexate & $5(19)$ \\
Mycophenolate & $3(12)$ \\
Azathioprine & $8(31)$ \\
RTX last cycle (months) & $28(9,50)$ \\
CYC last cycle (months) & $15(7,77)$ \\
Prednisolone & $11(42)$ \\
Prednisolone dose (mg daily) & $5(5,10)$ \\
Disease activity & \\
BVAS & $2(1,4)$ \\
Remission (BVAS & $1 \mathrm{n}(\%)]$ \\
VDI & \\
\hline
\end{tabular}

Median (25th, 75th percentile) values are presented unless stated otherwise

ANCA Anti-neutrophil cytoplasmic antibody, BMI body mass index, $B P$ blood pressure, $B V A S$ Birmingham Vasculitis Activity Score, $C V$ cardiovascular disease, $C R P$ C-reactive protein, $C Y C$ cyclophosphamide, ESR erythrocyte sedimentation rate, $R T X$ rituximab, VDI vasculitis damage index

\section{Discussion}

To our knowledge, this is the first prospective, multi-parametric CMR imaging study in GPA patients with no previous diagnosis of CVD or diabetes mellitus. As a feasibility study, it was not powered to detect significant associations between CMR outcomes and GPA disease characteristics, however we believe our results provide new insights into the prevalence and distribution of primary subclinical
CVD in GPA and align with our findings using similar CMR protocols in other IMID cohorts [38, 39].

Few studies have investigated cardiac involvement in GPA using CMR imaging. One retrospective, non-controlled study with $1.5 \mathrm{~T}$ CMR by the French Vasculitis Study Group [24] recruited over an 8-year period and did not comprehensively exclude concomitant CVD. Other investigators [25] performed a 1.5T CMR imaging with T1 and T2 mapping study in consecutive AAV patients and age and sex matched healthy volunteers. The authors reported a pooled analysis of AAV which included several patients with eosinophilic GPA (EGPA), a subset of AAV characterised by a distinct type of myocardial involvement. Current management guidelines of GPA do not recommend CV imaging at disease onset nor during follow up, although CVD is a major determinant of mortality in AAV [40], however the specific cardiac abnormalities associated with GPA have not been defined.

The primary finding of this feasibility study is the detection of myocardial fibrosis in approximately one-third of GPA patients, in line with previous reports [24, 25, 41]. LGE corresponds to areas of myocardial fibrosis as shown by comparison with histopathology [42], and its presence has prognostic value in ischaemic and non-ischaemic cardiomyopathies [43, 44]. The myocardial LGE can show different patterns according to diverse pathology; trans-mural LGE is typical of myocardial infarction whilst non-ischemic conditions associate with focal enhancement. Interestingly, the LGE seen in our GPA patients was focal and distributed subepicardially or at the mid-wall in most cases, in keeping with a non-ischaemic cause. Furthermore, this fibrotic tissue was frequently found in the lateral segments.

The presence of LGE was not associated with traditional CV risk factors, and we minimised the likelihood of underlying undiagnosed CAD by excluding GPA patients at highest risk (i.e. those with previous $\mathrm{CV}$ events and/or with DM). LGE was associated with PR3 ANCA negativity and also renal involvement, suggesting a potential relationship between myocardial fibrosis in GPA and specific disease characteristics. This finding is of clinical interest as the presence or absence of LGE on CMR could, in the future, help with CVD risk stratification in patients with GPA [19]. Whether myocardial LGE is associated with increased risk of relapse or disease severity needs to be addressed with further research.

Native T1 and ECV are indicative of a diffuse intramyocardial fibrosis/inflammation, including in the absence of infarction [45]. Native T1 and ECV values were slightly elevated in patients with GPA when compared to healthy volunteers, in keeping with previous reports [25]. We only included patients with established GPA, suggesting that a degree of cardiac inflammation is present throughout the GPA disease continuum, and, in our cohort, an association 
Table 2 Cardiovascular risk factors of study population

\begin{tabular}{llll}
\hline $\begin{array}{l}\text { Cardiovascular risk factors } \\
\text { and medications }\end{array}$ & GPA patients $(\mathrm{n}=26)$ & Heathy volunteers $(\mathrm{n}=25)$ & P value \\
\hline Age (years) & $58(45,66)$ & $61(51,66)$ & 0.665 \\
Female sex [n (\%)] & $12(46)$ & $7(28)$ & 0.180 \\
Smoking status (ever) & $5(19)$ & $0(0)$ & 0.021 \\
BMI [kg/m $\left.{ }^{2}\right]$ & $28.7(24.3,30.8)$ & $27.0(23.9,28.9)$ & 0.175 \\
Obese [n (\%)] & $9(35)$ & $5(20)$ & 0.242 \\
Hypertension [n (\%)] & $10(39)$ & $7(28)$ & 0.556 \\
SBP [mmHg] & $137(121,141)$ & $122(117,142)$ & 0.127 \\
DBP [mmHg] & $76(67,82)$ & $72(61,79)$ & 0.279 \\
MAP [mmHg] & $94(86,103)$ & $90(82,96)$ & 0.152 \\
Dyslipidaemia [n $(\%)]$ & $9(35)$ & NA & NA \\
\hline
\end{tabular}

Median (25th, 75th percentile) values are presented unless stated otherwise

$B M I$ body mass index, $B P$ blood pressure, $C V$ cardiovascular disease, $C R P$ C-reactive protein, $E S R$ erythrocyte sedimentation rate, $L V$ left ventricular, $M A P$ mean arterial pressure, $M C V$ mean corpuscular volume, $N A$ data not available between native T1 and vasculitis-related damage (VDI) and inflammation (CRP).

Assessment of patients with GPA using CMR may also reveal LV remodelling, with characteristics similar to that seen in our previously reported IMID cohorts [37, 38]. LV volumes were decreased in GPA patients compared to healthy volunteers, similar findings have been reported with echocardiography in RA patients, often in association with a concentric LV geometry and subclinical LV dysfunction [46-48]. Interestingly, LV mass in GPA patients was also significantly lower than controls and below published normal ranges [45]; which has been previously reported in patients with AAV [25] and also systemic sclerosis [39] and RA [38], implying the presence of inflammatory myocardial involvement across the IMID spectrum.

Cardiac abnormalities are common in GPA patients and the likelihood of cardiac damage may be highest in the early stages of the disease $[49,50]$. In this study we report primary, non-ischaemic myocardial abnormalities in asymptomatic GPA patients with well controlled disease, suggesting that subclinical cardiac pathology cay occur in GPA patients despite the absence of cardiac signs or symptoms [8]. The clinical implications of this remain to be determined. Additionally, in our study, a longer disease duration was associated with several measures of LV systolic dysfunction, underlining the importance of aiming for disease remission rather than low disease activity in GPA. In the future, disease activity indexes could be used to aid the prediction of future CV events and CVD-related mortality in patients with GPA [51].

\section{Clinical implications and novelties}

In this study, we prospectively demonstrated the feasibility and usefulness of CMR in GPA. Our data show that patients with GPA have both subclinical inflammation and fibrosis detected by CMR mapping techniques, despite being free of overt cardiac disease and vasculitic symptoms. ECV was associated with relapsing disease, suggesting that a clinically non-resolving vasculitis may cause active myocardial inflammation. Moreover, we demonstrated that myocardial fibrosis was present even when ANCA were not detectable in patients with skin and pulmonary involvement from vasculitis. Hence, our data could help to define those patients clinically at a higher risk of cardiac involvement. T1 mapping, ECV and LGE should all be assessed for the characterization of myocardial involvement in GPA, as they provide complementary information about diffuse myocardial involvement compared to one technique alone. Whether such alterations are reversible or can progress to additional damage should be addressed in further research.

\section{Study strengths and limitations}

Major strengths include: first, the pre-defined protocol with 3.0T CMR imaging, which informed rheumatologists to perform T1 mapping, LGE and ECV to detect myocardial involvement in GPA; second, the inclusion of controls also matched for arterial BP, which allowed to exclude hypertensive cardiac alterations; third, the multidisciplinary nature of our research group, which includes clinician-researchers experienced in both vasculitis and cardiac imaging; and last, we fully described the vasculitis status and characteristics and their associations with CMR abnormalities. Finally, our study included mostly patients with low disease activity (median BVAS $=2$ ), actually showing that subclinical myocardial involvement can be detected even in GPA patients without active significant organ involvement.

The most important limitation of our study is a small size, though it is comparable to previous research groups 
Table 3 Comparison of CMR characteristics between GPA patients and heathy volunteers

\begin{tabular}{|c|c|c|c|}
\hline CMR measures & GPA patients $(n=26)$ & Heathy volunteers $(\mathrm{n}=25)$ & $\mathrm{P}$ value \\
\hline \multicolumn{4}{|l|}{ LV volumes and mass } \\
\hline LV EDV index $\left(\mathrm{mL} / \mathrm{m}^{2}\right)$ & $69(61,84)$ & $79(70,89)$ & 0.105 \\
\hline LV ESV index $\left(\mathrm{mL} / \mathrm{m}^{2}\right)$ & $28(23,37)$ & $32(28,39)$ & 0.122 \\
\hline LV mass index $\left(\mathrm{g} / \mathrm{m}^{2}\right)$ & $37(42,47)$ & $50(42,56)$ & 0.014 \\
\hline LV mass/LVEDV, g/mL & $0.59(0.48,0.66)$ & $0.59(0.54,0.66)$ & 0.486 \\
\hline \multicolumn{4}{|l|}{ LV function } \\
\hline $\operatorname{LV}$ EF $(\%)$ & $59(56,63)$ & $58(53,62)$ & 0.351 \\
\hline \multicolumn{4}{|l|}{ LV peak systolic strain } \\
\hline Base & $-0.19(-0.21,-0.16)$ & $-0.21(-0.22,-0.19)$ & 0.017 \\
\hline Mid & $-0.21(-0.23,-0.20)$ & $-0.22(-0.23,-0.20)$ & 0.298 \\
\hline Apex & $-0.21(-0.23,-0.16)$ & $-0.23(-0.25,-0.20)$ & 0.101 \\
\hline Torsion $\left({ }^{\circ}\right)$ & $12.3(9.2,14.5)$ & $13.8(11.6,16.0)$ & 0.086 \\
\hline Peak twist ${ }^{\mathrm{a}}$ & $14.1(9.0,15.7)$ & $14.1(11.7,17.7)$ & 0.222 \\
\hline \multicolumn{4}{|l|}{ Myocardial tissue characterization } \\
\hline Native T1 (ms) & $1225(1199,1255)$ & $1203(1185,1233)$ & 0.006 \\
\hline Extracellular volume $(\%)^{\mathrm{b}}$ & $25.0(23.3,27.2)$ & $23.6(20.5,25.1)$ & 0.009 \\
\hline Increased extracellular volume [n (\%)] & $6 / 25(24)$ & $0(0)$ & 0.022 \\
\hline $\operatorname{LGE}[\mathrm{n}(\%)]^{\mathrm{b}}$ & $8 / 25(32)$ & $0 / 25(0)$ & 0.010 \\
\hline \multicolumn{4}{|l|}{ LGE distribution } \\
\hline Subepicardial & $4 / 8(50)$ & $0 / 25(0)$ & NA \\
\hline Midwall & $2 / 8(25)$ & $0 / 25(0)$ & NA \\
\hline RVIP & $2 / 8(25)$ & $0 / 25(0)$ & NA \\
\hline \multicolumn{4}{|l|}{ LGE segments } \\
\hline Basal antero-lateral & $4 / 8(50)$ & $0 / 25(0)$ & NA \\
\hline Basal infero-lateral & $3 / 8(38)$ & $0 / 25(0)$ & NA \\
\hline Basal antero-septal & $1 / 8(13)$ & $0 / 25(0)$ & NA \\
\hline LGE scar tissue (g) & $1.25(0.81,2.63)$ & $0(0,0)$ & NA \\
\hline \multicolumn{4}{|l|}{ Aortic distensibility } \\
\hline Descending aorta $\left(10^{-3} \mathrm{mmHg}^{-1}\right)$ & $2.95(2.29,3.64)$ & NA & NA \\
\hline Abnormal stiffness [n (\%)] & $19 / 26(73)$ & NA & NA \\
\hline
\end{tabular}

Median (25th, 75th percentile) values are presented unless stated otherwise

Left and right ventricle volumes are indexed by body surface area

$C M R$ cardiovascular magnetic resonance, $E C V$ extracellular-volume fraction, $E D V$ end-diastolic volume, $E S V$ end-systolic volume, $L G E$ late gadolinium enhancement, $L V$ left ventricular, $L V E F$ left ventricular ejection fraction, $N A$ data not available, $R V I P$ right ventricle insertion point

${ }^{a}$ Data available in 24/26 GPA patients

${ }^{\mathrm{b}}$ Data available in 25/26 GPA patients reporting on the same topic. We advocate multi-centric studies to increment the study population and to validate our results. Another limitation is that we could not entirely exclude CAD, yet we excluded a priori participants with overt CVD, diabetes or uncontrolled hypertension. Finally, a validation procedure, such as endomyocardial biopsy, was not performed. Endomyocardial biopsy was not offered to patients as they all had preserved LV-EF and were all asymptomatic for cardiac symptoms. However, endomyocardial biopsy has several limitations, including low sensibility, lowering its diagnostic benefit.

\section{Conclusion}

This study describes CMR imaging findings in patients with established stable GPA. Myocardial fibrosis and abnormal LV geometry were more common in GPA patients than in matched healthy controls, and correlate with certain disease characteristics. Although the prognostic implications of our results are yet to be fully understood, our group has reported on the frequency and severity of cardiac abnormalities in a population of GPA 

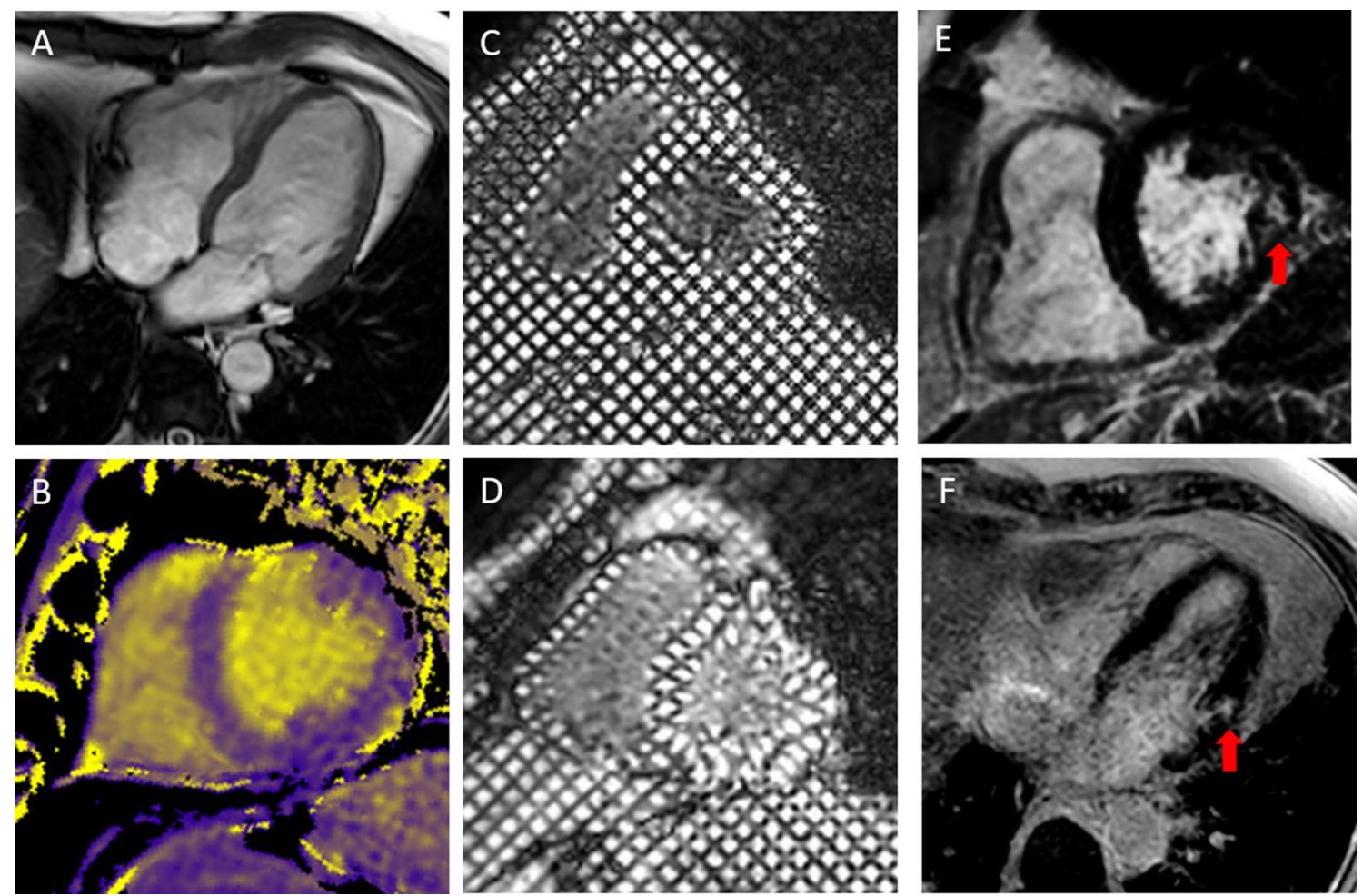

Fig. 2 CMR imaging from a single patient with GPA: SSFP cine imaging (a), Native T1 map with colour scale from $1000 \mathrm{~ms}$ (purple) to $2000 \mathrm{~ms}$ (yellow) (b), grid tags at end diastole (c) and end systole

patients which will inform future studies aimed at evaluating the management of cardiac involvement in patients with GPA.

Acknowledgements We wish to thank all patients participating in the study. We are grateful to Dr Andrew Barr and Dr Shouvik Dass for helping in the recruitment process, and to Catherine Bruckner for administrative and logistic support. The research is supported by the National Institute for Health Research (NIHR) infrastructure at Leeds. The views expressed are those of the author(s) and not necessarily those of the NHS, the NIHR or the Department of Health.

Authors' contributions Study concept and design: AG, MHB, JA, JG. Drafting the manuscript: AG. Revising the article and intellectual content: RBD, MHB, JA, JG, PS, JG, SP. Data collection: AG, RBD, PS. Analysis and interpretation of the data: AG, RBD, PS. AG, RBD, LAB, MHB, JA have full access to all data and take responsibility for the integrity of the data. All authors revised the paper and approved the final version.

Funding No specific funding was received from any bodies in the public, commercial or not-for-profit sectors to carry out the work described in this article. (d) and late gadolinium enhancement imaging in short (e) and long axis (f) with focal fibrosis shown by red arrow

\section{Compliance with ethical standards}

Conflict of interest All authors have nothing to declare.

Ethics approval The study was reviewed by an Independent Ethics Committee/Institutional Review Board. The study was conducted according to the Declaration of Helsinki, Good Clinical Practice.

Informed consent All patients gave their written, informed consent to take part in the study with approval of the National Research Ethics Service (10/H1306/88).

Open Access This article is licensed under a Creative Commons Attribution 4.0 International License, which permits use, sharing, adaptation, distribution and reproduction in any medium or format, as long as you give appropriate credit to the original author(s) and the source, provide a link to the Creative Commons licence, and indicate if changes were made. The images or other third party material in this article are included in the article's Creative Commons licence, unless indicated otherwise in a credit line to the material. If material is not included in the article's Creative Commons licence and your intended use is not permitted by statutory regulation or exceeds the permitted use, you will need to obtain permission directly from the copyright holder. To view a copy of this licence, visit http://creativecommons.org/licenses/by/4.0/. 


\section{References}

1. Yates M, Watts R (2017) ANCA-associated vasculitis. Clin Med (Lond) 17(1):60-64

2. Tan JA, Dehghan N, Chen W, Xie H, Esdaile JM, Avina-Zubieta JA (2017) Mortality in ANCA-associated vasculitis: ameta-analysis of observational studies. Ann Rheum Dis 76(9):1566-1574

3. Garen T, Lerang K, Hoffmann-Vold AM et al (2018) Mortality and causes of death across the systemic connective tissue diseases and the primary systemic vasculitides. Rheumatology 58:313-320

4. Berti A, Matteson EL, Crowson CS, Specks U, Cornec D (2018) Risk of cardiovascular disease and venous thromboembolism among patients with incident ANCA-associated vasculitis: a 20-year population-based cohort study. Mayo Clin Proc 93(5):597-606

5. Houben E, Penne EL, Voskuyl AE et al (2018) Cardiovascular events in anti-neutrophil cytoplasmic antibody-associated vasculitis: a meta-analysis of observational studies. Rheumatology (Oxford, England) 57(3):555-562

6. Avina-Zubieta JA, Mai A, Amiri N et al (2016) Risk of myocardial infarction and stroke in patients with granulomatosis with polyangiitis (Wegener's): a population-based study. Arthritis Rheum (Hoboken, NJ) 68(11):2752-2759

7. Solans-Laque R, Fraile G, Rodriguez-Carballeira M et al (2017) Clinical characteristics and outcome of Spanish patients with ANCA-associated vasculitides: impact of the vasculitis type, ANCA specificity, and treatment on mortality and morbidity. Medicine 96(8):e6083

8. Hazebroek MR, Kemna MJ, Schalla S et al (2015) Prevalence and prognostic relevance of cardiac involvement in ANCA-associated vasculitis: eosinophilic granulomatosis with polyangiitis and granulomatosis with polyangiitis. Int J Cardiol 199:170-179

9. Sherer Y, Shoenfeld Y (2006) Mechanisms of disease: atherosclerosis in autoimmune diseases. Nat Clin Pract Rheumatol 2(2):99-106

10. An X, Han Y, Zhang B et al (2017) Takayasu arteritis presented with acute heart failure: case report and review of literature. ESC Heart Fail 4(4):649-654

11. Bluett R, McDonnell D, O’Dowling C, Vaughan C (2017) Eosinophilic myocarditis as a first presentation of eosinophilic granulomatosis with polyangiitis (Churg-Strauss syndrome). BMJ Case Rep. https://doi.org/10.1136/bcr-2017-221227

12. Qiao L, Gao D (2016) A case report and literature review of Churg-Strauss syndrome presenting with myocarditis. Medicine 95(51):e5080

13. Ammirati E, Cipriani M, Musca F et al (2016) A life-threatening presentation of eosinophilic granulomatosis with polyangiitis. $\mathrm{J}$ Cardiovasc Med (Hagerstown) 17(Suppl 2):e109-e111

14. Bechman K, Gopalan D, Nihoyannopoulos P, Mason JC (2017) A cohort study reveals myocarditis to be a rare and life-threatening presentation of large vessel vasculitis. Sem Arthritis Rheum 47(2):241-246

15. Groh M, Masciocco G, Kirchner E et al (2014) Heart transplantation in patients with eosinophilic granulomatosis with polyangiitis (Churg-Strauss syndrome). J Heart Lung Transplant 33(8):842-850

16. Oliveira GH, Seward JB, Tsang TS, Specks U (2005) Echocardiographic findings in patients with Wegener granulomatosis. Mayo Clin Proc 80(11):1435-1440

17. Kuller LH, Shemanski L, Psaty BM et al (1995) Subclinical disease as an independent risk factor for cardiovascular disease. Circulation 92(4):720-726

18. Kuller LH, Arnold AM, Psaty BM et al (2006) 10-year follow-up of subclinical cardiovascular disease and risk of coronary heart disease in the Cardiovascular Health Study. Arch Intern Med 166(1):71-78

19. Fent GJ, Greenwood JP, Plein S, Buch MH (2017) The role of non-invasive cardiovascular imaging in the assessment of cardiovascular risk in rheumatoid arthritis: where we are and where we need to be. Ann Rheum Dis 76(7):1169-1175

20. Mavrogeni SI, Kitas GD, Dimitroulas T et al (2016) Cardiovascular magnetic resonance in rheumatology: current status and recommendations for use. Int J Cardiol 217:135-148

21. Hunold P, Schlosser T, Vogt FM et al (2005) Myocardial late enhancement in contrast-enhanced cardiac MRI: distinction between infarction scar and non-infarction-related disease. Am J Roentgenol 184(5):1420-1426

22. Iles L, Pfluger H, Phrommintikul A et al (2008) Evaluation of diffuse myocardial fibrosis in heart failure with cardiac magnetic resonance contrast-enhanced T1 mapping. J Am Coll Cardiol 52(19):1574-1580

23. Ferreira VM, Piechnik SK, Dall'Armellina E et al (2013) T(1) mapping for the diagnosis of acute myocarditis using CMR: comparison to T2-weighted and late gadolinium enhanced imaging. JACC Cardiovasc Imaging 6(10):1048-1058

24. Pugnet G, Gouya H, Puechal X et al (2017) Cardiac involvement in granulomatosis with polyangiitis: a magnetic resonance imaging study of 31 consecutive patients. Rheumatology (Oxford, England) 56(6):947-956

25. Greulich S, Mayr A, Kitterer D et al (2017) T1 and T2 mapping for evaluation of myocardial involvement in patients with ANCAassociated vasculitides. J Cardiovasc Magn Reson 19(1):6

26. Jennette JC, Falk RJ, Bacon PA et al (2013) 2012 revised International Chapel Hill consensus conference nomenclature of vasculitides. Arthritis Rheum 65(1):1-11

27. Mukhtyar C, Lee R, Brown D et al (2009) Modification and validation of the Birmingham vasculitis activity score (version 3 ). Ann Rheum Dis 68(12):1827-1832

28. Exley AR, Bacon PA, Luqmani RA et al (1997) Development and initial validation of the Vasculitis Damage Index for the standardized clinical assessment of damage in the systemic vasculitides. Arthritis Rheum 40(2):371-380

29. Schulz-Menger J, Bluemke DA, Bremerich J et al (2013) Standardized image interpretation and post processing in cardiovascular magnetic resonance: Society for Cardiovascular Magnetic Resonance (SCMR) Board of Trustees Task Force on Standardized Post Processing. J Cardiovasc Magn Reson 15(1):35

30. Plein S, Ridgway JP, Jones TR, Bloomer TN, Sivananthan MU (2002) Coronary artery disease: assessment with a comprehensive MR imaging protocol-initial results. Radiology 225(1):300-307

31. Kawel-Boehm N, Maceira A, Valsangiacomo-Buechel ER et al (2015) Normal values for cardiovascular magnetic resonance in adults and children. J Cardiovasc Magn Reson 17:29

32. Swoboda PP, McDiarmid AK, Erhayiem B et al (2017) Diabetes mellitus, microalbuminuria, and subclinical cardiac disease: identification and monitoring of individuals at risk of heart failure. $\mathbf{J}$ Am Heart Assoc 6(7):e005539

33. Dong Y, Sun J, Yang D et al (2018) Right ventricular septomarginal trabeculation hypertrophy is associated with disease severity in patients with pulmonary arterial hypertension. PLoS ONE 34(9): 1439-1449

34. Reiter U, Reiter G, Kovacs G et al (2017) Native myocardial T1 mapping in pulmonary hypertension: correlations with cardiac function and hemodynamics. Eur Radiol 27(1):157-166

35. Karakus G, Zencirci E, Degirmencioglu A, Guvenc TS, Unal Aksu H, Yildirim A (2017) Easily measurable, noninvasive, and novel finding for pulmonary hypertension: hypertrophy of the basal segment of septomarginal trabeculation of right ventricle. Echocardiography 34(2):290-295 
36. Hesselstrand R, Scheja A, Wuttge DM, Arheden H, Ugander M (2011) Enlarged right-sided dimensions and fibrosis of the right ventricular insertion point on cardiovascular magnetic resonance imaging is seen early in patients with pulmonary arterial hypertension associated with connective tissue disease. Scand J Rheumatol 40(2):133-138

37. Nitsche C, Kammerlander AA, Binder C et al (2019) Native T1 time of right ventricular insertion points by cardiac magnetic resonance: relation with invasive haemodynamics and outcome in heart failure with preserved ejection fraction. Eur Heart J Cardiovasc Imaging 21:683-691

38. Bissell L-A, Erhayiem B, Hensor EM et al (2016) THU0099 cardiovascular MR (CMR) evidence for reduced LV mass in rheumatoid arthritis (RA), suggesting pathology other than atherosclerosis for heart failure. Ann Rheum Dis 75(Suppl 2):215

39. Dumitru RB, Bissell L-A, Erhayiem B et al (2018) THU0390 Patients with systemic sclerosis develop focal fibrosis over time, and increased ecv diffuse fibrosis seen in poor prognostic group - a first longitudinal cardiac mri study. Ann Rheum Dis 77(Suppl 2):410

40. Guillevin L, Pagnoux C, Seror R et al (2011) The Five-Factor Score revisited: assessment of prognoses of systemic necrotizing vasculitides based on the French Vasculitis Study Group (FVSG) cohort. Medicine 90(1):19-27

41. Mavrogeni S, Sfikakis PP, Gialafos E et al (2014) Cardiac tissue characterization and the diagnostic value of cardiovascular magnetic resonance in systemic connective tissue diseases. Arthritis Care Res 66(1):104-112

42. Mahrholdt H, Wagner A, Judd RM, Sechtem U, Kim RJ (2005) Delayed enhancement cardiovascular magnetic resonance assessment of non-ischaemic cardiomyopathies. Eur Heart J 26(15):1461-1474

43. Weng Z, Yao J, Chan RH et al (2016) Prognostic value of LGECMR in HCM: a meta-analysis. JACC Cardiovasc Imaging 9(12):1392-1402

44. Ganesan AN, Gunton J, Nucifora G, McGavigan AD, Selvanayagam JB (2018) Impact of late gadolinium enhancement on mortality, sudden death and major adverse cardiovascular events in ischemic and nonischemic cardiomyopathy: a systematic review and meta-analysis. Int J Cardiol 254:230-237

45. Haaf P, Garg P, Messroghli DR, Broadbent DA, Greenwood JP, Plein S (2016) Cardiac T1 mapping and extracellular volume (ECV) in clinical practice: a comprehensive review. J Cardiovasc Magn Reson 18(1):89

46. Cioffi G, Viapiana O, Ognibeni F et al (2015) Prevalence and factors related to inappropriately high left ventricular mass in patients with rheumatoid arthritis without overt cardiac disease. J Hypertens 33(10):2141-2149

47. Cioffi G, Viapiana O, Ognibeni F et al (2017) Prognostic role of subclinical left ventricular systolic dysfunction evaluated by speckle-tracking echocardiography in rheumatoid arthritis. J Am Soc Echocardiogr 30(6):602-611

48. Midtbo H, Semb AG, Matre K, Kvien TK, Gerdts E (2017) Disease activity is associated with reduced left ventricular systolic myocardial function in patients with rheumatoid arthritis. Ann Rheum Dis 76(2):371-376

49. Mavrogeni S, Markousis-Mavrogenis G, Koutsogeorgopoulou L et al (2017) Cardiovascular magnetic resonance imaging pattern at the time of diagnosis of treatment naive patients with connective tissue diseases. Int J Cardiol 236:151-156

50. Ahn SS, Park ES, Jung SM, Song JJ, Park YB, Lee SW (2017) Echocardiographic features in patients with ANCA-associated vasculitis within 3 months before and after diagnosis. Clin Rheum 36(12):2751-2759

51. Bai YH, Li ZY, Chang DY, Chen M, Kallenberg CG, Zhao MH (2018) The BVAS is an independent predictor of cardiovascular events and cardiovascular disease-related mortality in patients with ANCA-associated vasculitis: a study of 504 cases in a single Chinese center. Semin Arthritis Rheum 47(4):524-529

Publisher's Note Springer Nature remains neutral with regard to jurisdictional claims in published maps and institutional affiliations. 